IOANNIS K. Argyros (Lawton, OK)

\title{
LOCAL CONVERGENCE THEOREMS FOR NEWTON'S METHOD FROM DATA AT ONE POINT
}

Abstract. We provide local convergence theorems for the convergence of Newton's method to a solution of an equation in a Banach space utilizing only information at one point. It turns out that for analytic operators the convergence radius for Newton's method is enlarged compared with earlier results. A numerical example is also provided that compares our results favorably with earlier ones.

1. Introduction. In this study, we are concerned with the problem of approximating a solution $x^{*}$ of an equation

$$
F(x)=0,
$$

where $F$ is sufficiently many times Fréchet-differentiable on an open, convex subset $D$ of a Banach space $X$, with values in a Banach space $Y$.

Newton's method

$$
x_{n+1}=x_{n}-F^{\prime}\left(x_{n}\right)^{-1} F\left(x_{n}\right) \quad\left(n \geq 0, x_{0} \in D\right)
$$

has been used to generate a sequence converging to $x^{*}$. There is an extensive literature on local and semilocal convergence theorems for Newton's method. We refer the reader to [1]-[9] and the references there for such results.

Here we introduce some local results for Newton's method, which enable us to obtain a convergence radius larger than in earlier results [5], [7]-[10]. That is, we obtain a wider range of initial choices $x_{0}$ than it was possible before. This information is important and also finds applications in step length

2000 Mathematics Subject Classification: 65B05, 47H17, 49D15, 65G99, 65J15, 65N30, $65 \mathrm{~N} 35$.

Key words and phrases: Banach space, Newton's method, Fréchet-differentiable/analytic operator, radius of convergence, simple zero. 
selection in predictor-corrector continuation procedures [4], [5], [7]-[10]. See also Remark 5 for other applications.

At the end of the study we provide a numerical example to show that indeed our results can provide a larger convergence radius than before.

2. Convergence analysis. We state the following local convergence theorem for Newton's method:

Theorem 1. Let $F: D \subseteq X \rightarrow Y$ be twice Fréchet-differentiable. Assume:

(a) there exists a simple zero $x^{*} \in D$ of $F$;

(b) there exists a constant $\ell \geq 0$ such that

$$
\left\|F^{\prime}\left(x^{*}\right)^{-1} F^{\prime \prime}(x)\right\| \leq \ell \quad(x \in D) ;
$$

(c)

$$
\bar{U}\left(x^{*}, r_{1}=\frac{2}{3 \ell}\right)=\left\{x \in X \mid\left\|x-x^{*}\right\| \leq r_{1}\right\} \subseteq D .
$$

Then Newton's method $\left\{x_{n}\right\}(n \geq 0)$ generated by (2) is well defined, remains in $\bar{U}\left(x^{*}, r_{1}\right)$ for all $n \geq 0$, and converges to $x^{*}$ provided that $x_{0} \in \bar{U}\left(x^{*}, r_{1}\right)$. Moreover, the following error bounds hold for all $n \geq 0$ :

$$
\left\|x_{n+1}-x^{*}\right\| \leq \frac{\ell}{2\left[1-\ell\left\|x_{n}-x^{*}\right\|\right]}\left\|x_{n}-x^{*}\right\|^{2} .
$$

Proof. It follows from (3) that there exists $D_{0} \subseteq D$ such that $F^{\prime}$ is $\ell$-Lipschitz on $D_{0}$, i.e.,

$$
\left\|F^{\prime}\left(x^{*}\right)^{-1}\left[F^{\prime}(x)-F^{\prime}(y)\right]\right\| \leq \ell\|x-y\| \quad\left(x \in D_{0}\right) .
$$

Without loss of generality we can assume $D_{0}=D$. The rest of the theorem follows exactly as in [9].

REMARK 1. In order for us to replace $\ell$ in Lipschitz conditions or as a bound on Fréchet derivatives in convergence theorems for Newton's method, assume $F$ is analytic on $D$, set

and

$$
\gamma(x)=\sup _{k>1}\left\|\frac{1}{k !} F^{\prime}(x)^{-1} F^{(k)}(x)\right\|^{1 /(k-1)} \quad(x \in D),
$$

$$
\gamma=\gamma\left(x^{*}\right)
$$

Moreover, assume that

$$
\bar{U}\left(x^{*}, r / \gamma\right) \subseteq D, \quad r \in[0,1 / \gamma) .
$$

Then, for all $x \in U\left(x^{*}, r\right), i=1,2, \ldots$, we get 
(9) $\left\|F^{\prime}\left(x^{*}\right)^{-1} F^{(i+1)}(x)\right\|=\left\|\sum_{k=0}^{\infty} \frac{1}{k !} F^{\prime}\left(x^{*}\right)^{-1} F^{(i+k+1)}\left(x^{*}\right)\left(x-x^{*}\right)^{k}\right\|$

$$
\begin{aligned}
& \leq \sum_{k=0}^{\infty}(k+i+1)(k+i) \gamma^{k+i}\left\|x-x^{*}\right\|^{k} \\
& =\gamma^{i} \sum_{k=0}^{\infty}(k+i+1)(k+i)\left(\gamma\left\|x-x^{*}\right\|\right)^{k} \\
& \leq \delta_{i+1} \equiv \frac{(i+1) ! \gamma^{i}}{(1-\gamma r)^{i+2}} .
\end{aligned}
$$

It follows from $(6)$ that $\ell$ can be replaced by $\delta_{2}(\gamma \neq 0)$. In this case the convergence condition is

$$
r \leq \frac{(1-\gamma r)^{3}}{3 \gamma}
$$

which becomes

$$
z^{3}-3 z^{2}+6 z-1 \leq 0, \quad z=r \gamma
$$

Solving (11) we finally deduce that Newton's method converges, provided that

$$
x_{0} \in \bar{U}\left(x^{*}, r_{2}\right) \subseteq D
$$

where

$$
r_{2}=\frac{.182269}{\gamma} \quad(\gamma \neq 0)
$$

Hence, we showed:

Theorem 2. Let $F: D \subseteq X \rightarrow Y$ be analytic, $x^{*}$ be as in Theorem 1, $\gamma$ and $r_{2}$ as defined by (8) and (12) respectively. Moreover, assume:

$$
\begin{gathered}
r_{2} \in(0,1 / \gamma), \\
x_{0} \in \bar{U}\left(x_{0}, r_{2}\right), \\
\bar{U}\left(x_{0}, r_{2}\right) \subseteq D .
\end{gathered}
$$

Then the conclusions of Theorem 1 for Newton's method hold with $\delta_{2}$ and $r_{2}$ replacing $\ell$ and $r_{1}$ respectively.

The following local convergence theorem was essentially proved, e.g., [2] or $[3]$.

Theorem 3. Let $F: D \subseteq X \rightarrow Y$ be an $(m+1)$-times $(m \geq 2$, an integer) Fréchet-differentiable operator and $x^{*}$ be as in Theorem 1. Assume that there exist nonnegative constants $\alpha_{j}, j=2, \ldots, m+1$, such that: 


$$
\begin{aligned}
\left\|F^{\prime}\left(x^{*}\right)^{-1} F^{(j)}\left(x^{*}\right)\right\| & \leq \alpha_{j}, & & j=2, \ldots, m, \\
\left\|F^{\prime}\left(x^{*}\right)^{-1} F^{(m+1)}(x)\right\| & \leq \alpha_{m+1} & & (x \in D) .
\end{aligned}
$$

Denote by $r_{3}$ the minimum positive zero, guaranteed to exist by Descartes' rule of signs, of the function $g$ given by

$$
g(r)=\beta_{m} r^{m}+\beta_{m-1} r^{m-1}+\ldots+\beta_{1} r+\beta_{0},
$$

where

$$
\begin{aligned}
\beta_{m} & =\frac{2 m+1}{(m+1) !} \alpha_{m+1}, \\
\beta_{i} & =\frac{i+(i+2) !(i+1)}{(m+1) ! m !} \alpha_{i+1}, \quad i=2, \ldots, m-1, \\
\beta_{1} & =\frac{3}{2} \alpha_{2}, \\
\beta_{0} & =-1 .
\end{aligned}
$$

Then Newton's method $\left\{x_{n}\right\}(n \geq 0)$ generated by (2) is well defined, remains in $\bar{U}\left(x^{*}, r_{3}\right)$ for all $n \geq 0$ and converges to $x^{*}$ provided that $x_{0} \in \bar{U}\left(x^{*}, r_{3}\right)$. Moreover, the following error bounds hold for all $n \geq 0$ :

$$
\left\|x_{n+1}-x^{*}\right\| \leq a_{n}\left\|x_{n}-x^{*}\right\|^{2},
$$

where

$$
\begin{aligned}
b_{n}= & \frac{m}{(m+1) !} \alpha_{m+1}\left\|x_{n}-x^{*}\right\|^{m-1} \\
& +\frac{(m-1) \alpha_{m}}{m !}\left\|x_{n}-x^{*}\right\|^{m-2}+\ldots+\frac{\alpha_{2}}{2 !}, \\
c_{n}= & 1-\alpha_{2}\left\|x_{n}-x^{*}\right\|-\ldots-\frac{\alpha_{m}}{(m-1) !}\left\|x_{n}-x^{*}\right\|^{m-1} \\
& -\frac{\alpha_{m+1}}{m !}\left\|x_{n}-x^{*}\right\|^{m}, \\
a_{n}= & \frac{b_{n}}{c_{n}} .
\end{aligned}
$$

REMARK 2. Note that condition (17) implies the weaker $\alpha_{m+1}$-Lipschitz condition used in the proof of Theorem 2 in [2].

REMARK 3. We can now argue as we did after Theorem 1 . Replace $\alpha_{m+1}$ in Theorem 3 by $\delta_{m+1}$ and denote by $r_{4}$ the minimum positive zero of the function $h$ defined as $g$ with $\delta_{m+1}$ replacing $\alpha_{m+1}$.

We proved: 
Theorem 4. Let $F: D \subseteq X \rightarrow Y$ be analytic, let $x^{*}, \alpha_{j}, j=2, \ldots, m$, be as in Theorem 3, and let $\gamma$ and $r_{4}$ be defined above. Moreover, assume:

$$
\begin{gathered}
r_{4} \in(0,1 / \gamma), \\
x_{0} \in \bar{U}\left(x^{*}, r_{4}\right), \\
\bar{U}\left(x^{*}, r_{4}\right) \subseteq D .
\end{gathered}
$$

Then the conclusions of Theorem 3 for Newton's method hold with $\delta_{m+1}$ and $r_{4}$ replacing $\alpha_{m+1}$ and $r_{3}$ respectively.

Remark 4. Note that condition (15) in Theorem 2 or condition (29) in Theorem 4 are automatically satisfied when $D=X$.

\section{Applications}

REMARK 5. As noted in [1]-[5], [8]-[10] our results can be used for projection methods such as Arnold's, the generalized minimum residual method (GMRES), the generalized conjugate residual method (GCR), and for combined Newton-like/finite-difference projection methods.

REMARK 6 . The results obtained here can also be used to solve equations of the form $F(x)=0$, where $F^{\prime}$ satisfies the autonomous differential equation $([4],[7])$

$$
F^{\prime}(x)=T(F(x))
$$

where $T: Y \rightarrow X$ is a known continuously sufficiently many times Fréchetdifferentiable operator. Since $F^{\prime}\left(x^{*}\right)=T\left(F\left(x^{*}\right)\right)=T(0)$,

$$
F^{\prime \prime}\left(x^{*}\right)=F^{\prime}\left(x^{*}\right) T^{\prime}\left(F\left(x^{*}\right)\right)=T(0) T^{\prime}(0)
$$

etc., we can apply the results obtained here without actually knowing the solution $x^{*}$ of equation (1).

We complete our study with such an example.

Example. Let $X=Y=\mathbb{R}, D=U(0,1)$, and define a function $F$ on $D$ by

$$
F(x)=e^{x}-1 .
$$

Then it can easily be seen that we can set $T(x)=x+1$ in (30).

Using (4), (6), (8), (12), (16)-(18), and (31) we obtain, for $m=2$,

$$
\begin{gathered}
\alpha_{2}=1, \quad \alpha_{3}=e, \quad \gamma=.5, \\
r_{1}=.245253, \quad r_{2}=.364538, \quad r_{3}=.411254048, \quad r_{4}=.3822432 .
\end{gathered}
$$

Hence, our results provide a wider choice for $x_{0}$ than the corresponding ones in [9], [10, Theorem 3.1, p. 585]. This observation is important and also finds applications in step length selection in predictor-corrector continuation procedures [5], [8], [10]. 


\section{References}

[1] I. K. Argyros, Improved error bounds for Newton-like iterations under Chen-Yamamoto assumptions, Appl. Math. Lett. 10 (1997), no. 4, 97-100.

[2] -, Local convergence theorems for Newton's method using outer or generalized inverses and $m$-Fréchet differentiable operators, Math. Sci. Res. Hot-Line 4 (2000), no. $4,47-56$.

[3] -, A Newton-Kantorovich theorem for equations involving m-Fréchet differentiable operators and applications in radiative transfer, J. Comput. Appl. Math. 131 (2001), 149-159.

[4] I. K. Argyros and F. Szidarovszky, The Theory and Applications of Iteration Methods, CRC Press, Boca Raton, FL, 1993.

[5] P. N. Brown, A local convergence theory for combined inexact-Newton/finite-difference projection methods, SIAM J. Numer. Anal. 24 (1987), 407-434.

[6] J. M. Gutiérrez, A new semilocal convergence theorem for Newton's method, J. Comput. Appl. Math. 79 (1997), 131-145.

[7] L. V. Kantorovich and G. R. Akilov, Functional Analysis, Pergamon Press, New York, 1982.

[8] F. A. Potra, On Q-order and R-order of convergence, J. Optim. Theory Appl. 63 (1989), 415-431.

[9] W. C. Rheinboldt, An adaptive continuation process for solving systems of nonlinear equations, in: Banach Center Publ. 3, PWN, Warszawa, 1977, 129-142.

[10] T. J. Ypma, Local convergence of inexact Newton methods, SIAM J. Numer. Anal. 21 (1984), 583-590.

Department of Mathematics

Cameron University

Lawton, OK 73505, U.S.A.

E-mail: ioannisa@cameron.edu

Received on 23.11.2001;

revised version on 13.6.2002 